\title{
Erratum to: From 2000 to 2016: Which Second-Line Treatment in Advanced Non-Small Cell Lung Cancer?
}

Ettore $D^{\prime}$ Argento, $M D, P h D^{1}$

Sabrina Rossi, $M D^{2, *}$

Giovanni Schinzari, MD, PhD ${ }^{1}$

Antonia Strippoli, MD, $P h D^{1}$

Michele Basso, MD, PhD ${ }^{1}$

Alessandra Cassano, MD, PhD ${ }^{1}$

Carlo Barone, MD, $P h D^{1}$

\section{Address}

${ }^{1}$ Department of Medical Oncology, Catholic University of Sacred Heart, Largo A. Gemelli, 8, 00168, Rome, Italy

*,2Department of Medical Oncology, Humanitas Clinical and Research Center, Via Manzoni, 56, 20089, Rozzano, MI, Italy

Email:sbm.rossi85@gmail.com

Published online: 20 December 2016

(C) Springer Science+Business Media New York 2016

Erratum to: Curr Treat Options in Oncol (2016) 17:59

DOI 10.1007/s11864-016-0437-x

In the original version of this article, which published in Current Treatment Options in Oncology, Volume 17, Issue 12, December 2016, the columns within the second page of Table 2 became disoriented, rendering the table inaccurate. The original article has been rectified, and the correct Table 2 is provided here.

The online version of the original article can be found at http://dx.doi.org/10.1007/s11864-016-0437-x. 
Table 2. Main characteristics of trials studying new anti-angiogenic and immunotherapy agents

\begin{tabular}{|c|c|c|c|c|}
\hline & $\begin{array}{l}\text { Docetaxel + } \\
\text { Nintedanib } \\
{[6 \bullet \bullet]}\end{array}$ & $\begin{array}{l}\text { Docetaxel + } \\
\text { Ramucirumab } \\
{[7 \bullet \bullet]}\end{array}$ & $\begin{array}{l}\text { Nivolumab } \\
{[8 \bullet \bullet, 9 \bullet \bullet]}\end{array}$ & $\begin{array}{l}\text { Pembrolizumab } \\
{[10 \bullet \bullet, 33 \bullet \bullet]}\end{array}$ \\
\hline Phase & III & III & III $[8 \bullet \bullet-9 \bullet \bullet]$ & I, non randomized \\
\hline $\begin{array}{l}\text { Main eligibility } \\
\text { criteria }\end{array}$ & $\begin{array}{l}\text { Stage IIIb-IV NSCLC } \\
\text { pretreated with } \\
\text { one chemotherapy } \\
\text { regimen }\end{array}$ & $\begin{array}{l}\text { Stage IV NSCLC } \\
\text { pretreated with } \\
\text { first-line } \\
\text { platinum-based } \\
\text { chemotherapy }\end{array}$ & $\begin{array}{l}\text { Stage IIIB/IV sqNSCLC } \\
{[8 \bullet \bullet] \text { and nsqNSCLC }} \\
{[9 \bullet \bullet] \text { pretreated with a }} \\
\text { first-line } \\
\text { platinum-based regimen }\end{array}$ & $\begin{array}{l}\text { Advanced NSCLC }[10 \bullet \bullet] \\
\text { Stage IV NSCLC pretreated } \\
\text { with first-line platinum- } \\
\text { based chemotherapy/TKIs; } \\
\text { PD-L1 } \geq 1 \%[33]\end{array}$ \\
\hline $\begin{array}{l}\text { Main exclusion } \\
\text { criteria }\end{array}$ & $\begin{array}{l}\text { - Prior treatment } \\
\text { with docetaxel } \\
\text { - Active brain or } \\
\text { leptomeningeal } \\
\text { metastases } \\
\text { - Cavitary or necrotic } \\
\text { tumors } \\
\text { - Vascular invasion } \\
\text { - Major thrombotic or } \\
\text { bleeding events } \\
\text { (hereditary or not) } \\
\text { - Significant } \\
\text { cardiovascular } \\
\text { disease } \\
\text { - Anticoagulation or } \\
\text { antiplatelet therapy }\end{array}$ & $\begin{array}{l}\text { - Prior treatment } \\
\text { only with TKIs. } \\
\text { - Active brain } \\
\text { metastases } \\
\text { - Cavitary tumors } \\
\text { - Vascular invasion } \\
\text { - Major thrombotic } \\
\text { or bleeding events } \\
\text { (hereditary or } \\
\text { not) } \\
\text { - Significant } \\
\text { cardiovascular } \\
\text { disease } \\
\text { - Anticoagulation } \\
\text { therapy } \\
\text { antiplatelet } \\
\text { therapy }\end{array}$ & $\begin{array}{l}\text { - Untreated brain } \\
\text { metastases } \\
\text { - Leptomeningeal disease } \\
\text { - active, known or suspected } \\
\text { autoimmune disease } \\
\text { - Interstitial lung disease } \\
\text { - Systemic treatment with } \\
\text { corticosteroids or } \\
\text { immunosuppressive } \\
\text { medications [8-9] }\end{array}$ & $\begin{array}{l}\text { - Previous pneumonitis } \\
{[10 \bullet \bullet, 33]} \\
\text { - Active autoimmune disease } \\
\text { - Immunosuppressive therapy } \\
{[10 \bullet \bullet, 33]} \\
\text { - Previous treatment with } \\
\text { PD-1 checkpoint inhibitors } \\
\text { or docetaxel [33] } \\
\text { - Active brain metastases or } \\
\text { carcinomatous meningitis } \\
\text { [33] }\end{array}$ \\
\hline Control arm & Docetaxel & Docetaxel & Docetaxel $[8 \bullet \bullet]-[9 \bullet \bullet]$ & $\begin{array}{l}{[10 \bullet \bullet]} \\
\text { Docetaxel [33] }\end{array}$ \\
\hline $\begin{array}{l}\mathrm{N}^{\circ} \text { of enrolled } \\
\text { patients }\end{array}$ & 1314 & 1253 & $\begin{array}{l}260[8 \bullet \bullet] \\
582[9 \bullet \bullet]\end{array}$ & $\begin{array}{l}495[10 \bullet \bullet] \\
1034[33]\end{array}$ \\
\hline$\%$ of nsqNSCLC & $\begin{array}{l}49.2 \% \text { in } \\
\text { experimental arm }\end{array}$ & $\begin{array}{l}74 \% \text { in } \\
\text { experimental } \\
\text { arm }\end{array}$ & Inclusion criteria in $[9 \bullet \bullet]$ & $\begin{array}{l}81.0 \%[10 \bullet \bullet] \\
78.5 \%[33]\end{array}$ \\
\hline$\%$ of sqNSCLC & $\begin{array}{l}42.1 \% \text { in } \\
\quad \text { experimental arm }\end{array}$ & $\begin{array}{l}25 \% \text { in } \\
\text { experimental } \\
\text { arm }\end{array}$ & Inclusion criteria in $[8 \bullet \bullet]$ & $\begin{array}{l}17.2 \%[10 \bullet \bullet] \\
21.5 \%[33]\end{array}$ \\
\hline PFS/TTP & $\begin{array}{r}3.4 \text { vs. } 2.7 \mathrm{~m} \\
(p=.0019)\end{array}$ & $\begin{array}{l}4.5 \text { vs. } 3.0 \mathrm{~m} \\
\quad(p<.0001)\end{array}$ & $\begin{array}{l}3.5 \text { vs. } 2.8(p<.001)[8 \bullet \bullet] \\
2.3 \text { vs. } 4.2 \mathrm{~m}(p=.39)[9 \bullet \bullet]\end{array}$ & 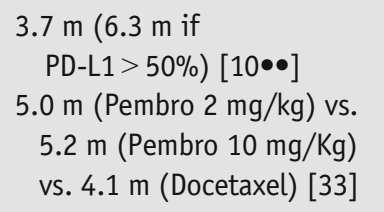 \\
\hline $\begin{array}{l}\text { OS/median } \\
\text { survival }\end{array}$ & $\begin{array}{l}\text { In all population: } \\
10.1 \text { vs. } 9.1 \mathrm{~m} \\
\quad(p=.2720) \\
\text { In adenocarcinoma: } \\
12.6 \text { vs. } 10.3 \mathrm{~m} \\
\quad(p=.0359)\end{array}$ & $\begin{array}{l}10.5 \text { vs } 9.1 \mathrm{~m} \\
\quad(p=.023)\end{array}$ & $\begin{array}{l}9.2 \text { s. } 6.0 \mathrm{~m}(p<.001) \\
\quad[8 \bullet \bullet] \\
12.2 \text { vs. } 9.4 \mathrm{~m}(p=.002) \\
\quad[9 \bullet \bullet]\end{array}$ & $\begin{array}{l}12.5 \mathrm{~m} \text { (Not reached if } \\
\text { PD-L1>50\%) [10••] } \\
10.4 \mathrm{~m} \text { (Pembro } \\
2 \mathrm{mg} / \mathrm{kg}) \text { vs. } 12.7 \mathrm{~m} \\
\text { (Pembro } 10 \mathrm{mg} / \mathrm{Kg}) \mathrm{vs} . \\
8.5 \mathrm{~m} \text { (Docetaxel) [33] }\end{array}$ \\
\hline
\end{tabular}


Table 2. (Continued)

\begin{tabular}{|c|c|c|c|c|}
\hline & $\begin{array}{l}\text { Docetaxel + } \\
\text { Nintedanib } \\
{[6 \bullet \bullet]}\end{array}$ & $\begin{array}{l}\text { Docetaxel }+ \\
\text { Ramucirumab } \\
{[7 \bullet \bullet]}\end{array}$ & $\begin{array}{l}\text { Nivolumab } \\
{[8 \bullet \bullet, 9 \bullet \bullet]}\end{array}$ & $\begin{array}{l}\text { Pembrolizumab } \\
{[10 \bullet \bullet, 33 \bullet \bullet]}\end{array}$ \\
\hline RR & $\begin{array}{l}4.4 \% \text { vs. } 3.3 \% \\
\quad(p=.31)\end{array}$ & $\begin{array}{l}23 \% \text { vs. } 14 \% \\
\quad(p<.0001)\end{array}$ & $\begin{array}{l}20 \% \text { vs. } 9 \%(p=.008)[8 \bullet \bullet] \\
19 \% \text { vs. } 12 \%(p=.002) \\
\quad[9 \bullet \bullet]\end{array}$ & $\begin{array}{l}19.4 \%(45.2 \% \text { if PD-L1 }> \\
50 \%)[10 \bullet \bullet \\
30 \% \text { (Pembro } 2 \mathrm{mg} / \mathrm{kg}) \text { vs. } \\
29 \% \text { (Pembro } 10 \mathrm{mg} / \mathrm{Kg}) \\
\text { vs. } 8 \% \text { (Docetaxel) }[33]\end{array}$ \\
\hline Time to response & - & - & $\begin{array}{l}2.2 \text { vs. } 2.1 \mathrm{~m}[8 \bullet \bullet] \\
2.1 \text { vs. } 2.6 \mathrm{~m}[9 \bullet \bullet]\end{array}$ & $\begin{array}{l}{[10 \bullet \bullet]} \\
9 \text { weeks for all subgroups } \\
{[33]}\end{array}$ \\
\hline $\begin{array}{r}\text { Duration of } \\
\text { response }\end{array}$ & - & - & $\begin{array}{l}\text { Not reached in nivolumab } \\
\text { arm }[8 \bullet \bullet] \\
17.2 \text { vs. } 5.6 \mathrm{~m}[9 \bullet \bullet]\end{array}$ & $\begin{array}{l}12.5 \mathrm{~m}[10 \bullet \bullet] \\
\text { Not reached for } \\
\text { pembrolizumab arms } \\
\text { [33] }\end{array}$ \\
\hline Markers & - & - & - & $\begin{array}{l}\text { PD-L1> 50\% }[10 \bullet \bullet] \\
\text { PD-L1+ }(\geq 1 \%)[33]\end{array}$ \\
\hline $\begin{array}{l}\text { \% G3-G4 adverse } \\
\text { events }\end{array}$ & $\begin{array}{l}\text { G3 } 7.4 \% \text { vs. } 8.9 \% \\
\text { G4 } 7.5 \% \text { vs. } 6.0 \%\end{array}$ & $79 \%$ vs. $71 \%$ & $\begin{array}{l}7 \% \text { vs. } 55 \%[8 \bullet \bullet] \\
10 \% \text { vs. } 54 \%[9 \bullet \bullet]\end{array}$ & $\begin{array}{l}9.5 \%[10 \bullet \bullet] \\
135(2 \mathrm{mg} / \mathrm{Kg}) \text { vs } 16 \% \\
\quad(10 \mathrm{mg} / \mathrm{kg}) \text { vs } 35 \% \\
\text { (docetaxel) }\end{array}$ \\
\hline $\begin{array}{l}\text { Most frequent } \\
\text { adverse } \\
\text { events in } \\
\text { experimental } \\
\text { arm }\end{array}$ & $\begin{array}{l}\text { Diarrhea, increase } \\
\text { in alanine and } \\
\text { aspartate } \\
\text { aminotransferase, } \\
\text { vomiting }\end{array}$ & $\begin{array}{l}\text { Neutropenia and } \\
\text { febrile } \\
\text { neutropenia, } \\
\text { bleeding, } \\
\text { epistaxis, } \\
\text { hypertension }\end{array}$ & $\begin{array}{l}\text { Fatigue, decreased } \\
\text { appetite, asthenia, } \\
\text { nausea } \\
{[8 \bullet \bullet, 9 \bullet \bullet]}\end{array}$ & $\begin{array}{l}\text { Fatigue, pruritus, decreased } \\
\text { appetite (hypothyroidism, } \\
\text { pneumonitis between } \\
\text { immunorelated AE) } \\
{[10 \bullet \bullet, 33]}\end{array}$ \\
\hline $\begin{array}{l}\text { Favourable } \\
\text { patients' } \\
\text { characteristics }\end{array}$ & $\begin{array}{l}\text { Adenocarcinoma } \\
\text { Never-smoker } \\
\text { Early progressive } \\
\quad \text { disease }\end{array}$ & Platinum-resistant & $\begin{array}{l}\leq 75 \text { years old }[8 \bullet \bullet] \\
\text { KRAS mutation }[9 \bullet \bullet] \\
\text { PD-L1 positive tumors }[9 \bullet \bullet] \\
\text { Current/former smokers }[9 \bullet \bullet] \\
\text { EGFR wild type }[9 \bullet \bullet]\end{array}$ & 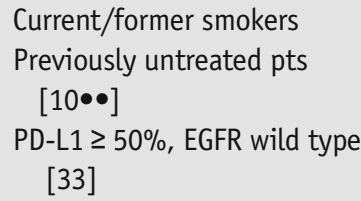 \\
\hline
\end{tabular}

Abbreviations: $w$ weeks, $m$ months, nsqNSCLC nonsquamous non-small-cell lung cancer, sqNSCLC squamous non-small-cell lung cancer, PFS/TTP progression free survival/time to progression, OS overall survival, $R R$ response rate 